\title{
Priming of pop-out: II. The role of position
}

\author{
VERA MALJKOVIC and KEN NAKAYAMA \\ Harvard University, Cambridge, Massachusetts
}

\begin{abstract}
In an earlier paper (Maljkovic \& Nakayama, 1994) we showed that repetition of an attention-driving feature primes the deployment of attention to the same feature on subsequent trials. Here we show that repetition of the target position also primes subsequent trials. Position priming shows a characteristic spatial pattern. Facilitation occurs when the target position is repeated on subsequent trials, and inhibition occurs when the target falls on a position previously occupied by a distractor. Facilitation and inhibition also exist, though somewhat diminished, for positions adjacent to those of the target and distractors. Assessing the effect of a single trial over time, we show that the characteristic memory trace exerts its strongest influence on immediately following trials and decays gradually over the succeeding, approximately five to eight, trials. Throughout this period, target-position facilitation is always stronger than distractor-position inhibition. The characteristics of position priming are also seen under conditions in which the attention-driving feature either stays the same or differs from the previous trial, suggesting that feature and position priming operate independently. In a separate experiment, using the fact that position priming is cumulative over trials, we show that position priming is largely object- or landmark-centered.
\end{abstract}

In an earlier companion paper, we reported that repeating odd target colors in a pop-out task speeds subsequent attentional deployment to same-color stimuli (Maljkovic \& Nakayama, 1994). The finding was revealed in a visual search task initially developed by Bravo and Nakayama (1992). In that paradigm, the observer must direct his or her focal attention to the odd target and respond to the target's subtle shape differences. The pop-out feature and the response feature are dissociated from each other and the priming is observed only when the feature, but not the response, is repeated. In the present paper we continue to explore the characteristics of the priming of pop-out, focusing now on the effect of stimulus position.

We first summarize the findings of feature priming reported in Maljkovic and Nakayama (1994). We conceive of the visual search task as a two-step process (see Bravo \& Nakayama, 1992), with a controlling stimulus property associated with each step. The first step, the attentiondriving feature - say, color-determines where attention is to be deployed. The second step requires focusing attention on a subtle aspect of the shape-which side of the target diamond is chopped off - which in turn determines the manual response, pressing the right or the left button. In the first step, the observer is required to find the odd "pop-out" target and, in the second step, to identify a subtle aspect in the shape of the target.

A memory trace of the attention-driving feature is laid down with each trial, and this trace facilitates deployment of attention to the same feature on the subsequent five to

This work was supported by AFOSR Grant F49620-92-J-0016 to K.N. We thank Barbara Gillam and Mary Potter for their comments on an earlier version of the manuscript. Correspondence should be addressed to K. Nakayama, Department of Psychology, Harvard University, 33 Kirkland St., Cambridge, MA 02138 (e-mail: ken@wjh.harvard.edu). eight trials, thereby priming the pop-out or attentional deployment (Maljkovic \& Nakayama, 1994). In the earlier paper we detailed the mechanism underlying this phenomenon, showing that the memory that alters performance exists for both target and distractor features. The target color of preceding trials positively primes the current same-color target, whereas the distractor color of preceding trials negatively primes the current same-color target. Repetition of the shape (and the concomitant repetition of the correct manual response) produces essentially no priming. ${ }^{.}$Thus, priming is highly selective. Certain seemingly obvious aspects of the previous trial leave no systematic residue. A similar detailed pattern of results was obtained in a version of the experiment in which spatial frequency was the attention-driving feature and the shape task was determining the vernier offset of an odd spatial-frequency patch.

A third property of both target and distractors is position. Because the position of the target is randomized between trials and uncorrelated with the attention-driving and shape variables, we might expect it to be irrelevant, for either the deployment of attention or the correct response. Yet the demand of the shape-recognition task requires that focal attention be directed to the position in space where the target is located. There thus remains the possibility that the visual system will not be indifferent to the effects of the previous position.

In trial-to-trial paradigms similar to ours, both facilitation (Kowler, Martins, \& Pavel, 1984; Neill, Terry, \& Valdes, 1994; Park \& Kanwisher, 1994) and inhibition to the repeated target position, the so-called inhibition of return, have been observed (Kwak \& Egeth, 1992; Maylor \& Hockey, 1985; Posner, Cohen, Choate, Hockey, \& Maylor, 1984). We might expect, therefore, that pop-out can be primed by the repetition of target position. But 
should we expect a facilitation or inhibition? There is reason to expect both. Our task involves focusing the attention sufficiently well to perform a high-acuity task at a location unpredictable from one trial to the next. This is similar to the tasks that produce facilitation (see, e.g., Kowler et al., 1984). On the other hand, if we consider popout an act of externally controlled orienting, we might expect inhibition (Maylor, 1985). Paying attention to a previously ignored location also produces an effect. In this case, the observer is slowed down, a phenomenon that has come to be known as "negative priming" (Park \& Kanwisher, 1994; Shapiro \& Loughlin, 1993; Tipper, Brehaut, \& Driver, 1990; Tipper, Weaver, Cameron, Brehaut, $\&$ Bastedo, 1991). We are therefore left to explore whether or not the repetition of the stimulus position can prime pop-out, whether there will be separate effects for target and distractor positions, and whether priming of target positions will be positive or negative.

\section{EXPERIMENT 1 \\ Influence of a Single-Trial Exposure Over Time: The Two-Position Case}

In this paper we use the visual search task described previously (Maljkovic \& Nakayama, 1994), in which observers judge which side of an odd-colored diamond is cut off. We show that given an equal probability of red and green targets (and opposite-color distractors), repetition of target and distractor colors leads to faster responses, whereas switching target and distractor colors leads to slower responses. The priming effect of a single trial is strongest for the next trial and then decays over the subsequent five to eight trials (Maljkovic \& Nakayama, 1994). In this first experiment, we examine what happens when target position is equally likely to be repeated or changed over successive trials by examining in detail the effect of a single trial over time. We use two target positions (left/ right), two colors (either red/green, or orange yellow/ocher yellow), and two responses (left/right). Each of these variables is completely unpredictable from trial to trial (Bernoulli sequences), and each is independent of the other.

\section{Method}

Subjects. A naive observer, E.F.B., and one of the authors, V.M., a practiced observer, participated in this experiment. ${ }^{2}$

Stimuli. The elements were red and green diamonds for $\mathrm{Ob}$ server E.F.B., and orange-yellow and ocher-yellow diamonds for Observer V.M. The single target was one color and the two distractors were the other. The colors were close to equiluminant, chosen so that reaction times (RTs) for each were comparable. Red had a luminance of $2.0 \mathrm{~cd} / \mathrm{m}^{2}$ and CIE coordinates $.612 / .351$; green had a luminance of $2.1 \mathrm{~cd} / \mathrm{m}^{2}$ and CIE coordinates $.313 / .553$; orange yellow had a luminance of $8.2 \mathrm{~cd} / \mathrm{m}^{2}$ and CIE coordinates $.541 / .411$, and the ocher yellow had a luminance of $8.6 \mathrm{~cd} / \mathrm{m}^{2}$ and CIE coordinates $.484 / .453$. The background was essentially black, with a luminance of $.1 \mathrm{~cd} / \mathrm{m}^{2}$. The diamonds were approximately $1.0^{\circ} \times$ $1.0^{\circ}$, with $0.14^{\circ}$ cut off either at the left or the right. They were arranged on a nearly circular ellipse with the major and the minor axes of $10.0^{\circ}$ and $8.1^{\circ}$, respectively. ${ }^{3}$ If the face of a clock were superimposed over the ellipse, the target could be at either 2 o'clock or 10 o'clock, with one distractor always at the 10 o'clock or 2 o'clock, respectively, and the other distractor always at 6 o'clock. Figure 1 shows a schematic representation of this layout. (Note that while the target could only be in one of two positions, the observer had to attend to the entire display in order to decide which was the oddcolored target.) The white fixation point, at the center of the ellipse, was $.32^{\circ} \times .37^{\circ}$ and had a luminance of $13.2 \mathrm{~cd} / \mathrm{m}^{2}$.

Procedure. When the stimulus appeared on the screen, the observer's task was to find the odd-colored target and respond by indicating which side of the target was cut off. From trial to trial, the position, color, and shape of the target were varied randomly and independently of one another. The stimulus stayed on the screen until the observer responded by pressing the left or right button of the mouse with the index and middle finger, respectively. The response was followed by a blank screen for 2 to $2.5 \mathrm{sec}$, after which a new stimulus was shown. The fixation point remained on at all times. Observers were instructed to fixate throughout the trial and to respond as fast as they could while trying to keep their error rate reasonably low. Observer E.F.B. ran 4,000 trials in blocks of 200 (in four sessions of 1,000 trials each); Observer V.M. ran 3,400 trials in blocks of 200 (in two sessions of 1,000 and one of 1,400 trials). ${ }^{4}$

\section{Results and Discussion}

Our major finding was that previous trials positively primed subsequent trials having the same target position. Thus, if attention was directed to a specific locus in the recent past, it will be easier to direct it to that locus in the future. The effect lasts for approximately five to eight trials.

To show a comprehensive picture of this priming, we used a new method of data analysis, introduced in Maljkovic and Nakayama (1994). The method, "memory kernel analysis," is analogous to the nonlinear analysis employed in electrophysiological research in that it measures pair-wise interactions between events at different time intervals (Sutter, 1992). It partitions RTs for a given trial in the past into cases in which the position was the same as or different from that of the current trial. To obtain an estimated baseline of noise or variability of the method, it also analyzes pairs that correspond to future trials. These

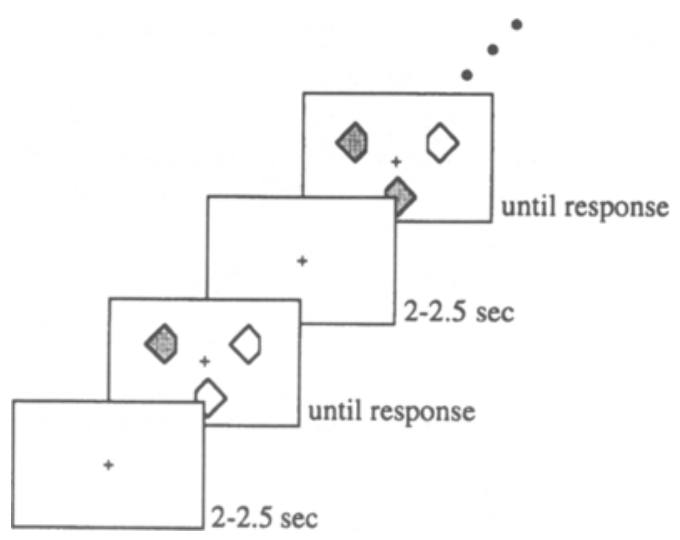

Figure 1. Schematic representation of the procedure described in Experiment 1. The task is to respond to the side on which the odd-colored diamond is cut off. The target position switches randomly between left and right from trial to trial (it never appears at the bottom). Target and distractor colors switch unpredictably from trial to trial. (The stimulus array is not shown to scale; stimuli are shown larger, for clarity.) 
pairs, of course, should have no influence on the current trial because they have not yet occurred.

A target $i$ trial in the past could have appeared in the same or in the different position as the target on the current trial. What is plotted in Figure 2 are correct RTs (on $y$-axis) for trial $n$ given the same or different target position on Trial $n-i$ (filled circles represent the same position, open triangles the different position). ${ }^{5}$ Note that in this method of analysis the influence of trials between $n-i$ and $n$ are averaged out, because over a large number of trials there is the same number of same and different positions for each in-between trial. The gray square at the intersection of the horizontal and vertical lines is the overall mean and represents the baseline against which we can compare the influence of each trial. The influence of past trials is shown to the left of the vertical line, and the influence of future trials is to the right of this line. Although future trials could have had no influence on the current trial, they provide an additional baseline-intrinsic RT variability not due to a memory trace.

A clear effect of repeated and changed position is shown in Figure 2. When the current trial had the same target position as recent trials, RTs were shorter; when they had a different target position, they were longer. Further, these differences were the strongest for trials just preceding the current trial, diminishing over the next five to eight trials. This temporal pattern (with one subtle difference to be discussed below) is essentially the same as that observed in color priming (Maljkovic \& Nakayama, 1994).

The clear facilitation found with repetition of the target position, from the previous trial as well as from each of the five to eight trials prior to the current trial, is surprising. It indicates that performance on a current trial is unexpectedly dependent on whether a target position matched that of a single previous trial, many trials back. A single trial therefore affects subsequent trials for approximately $30 \mathrm{sec}$ given our response-trial interval of $2-2.5 \mathrm{sec}^{6}$ The position priming exists in addition to the feature priming (color, spatial frequency), and has a similar time course.

The results show no obvious evidence of "inhibition of return," that is, increased RTs for the previously attended location. However, as readers may have noticed, facilitation is not the greatest for Trial $n-1$, but for Trial $n-2$. In our study of feature priming (Maljkovic \& Nakayama, 1994) we never found such nonmonotonicity in the decay of priming. The greatest effect on the current trial was always exerted by the immediately previ-

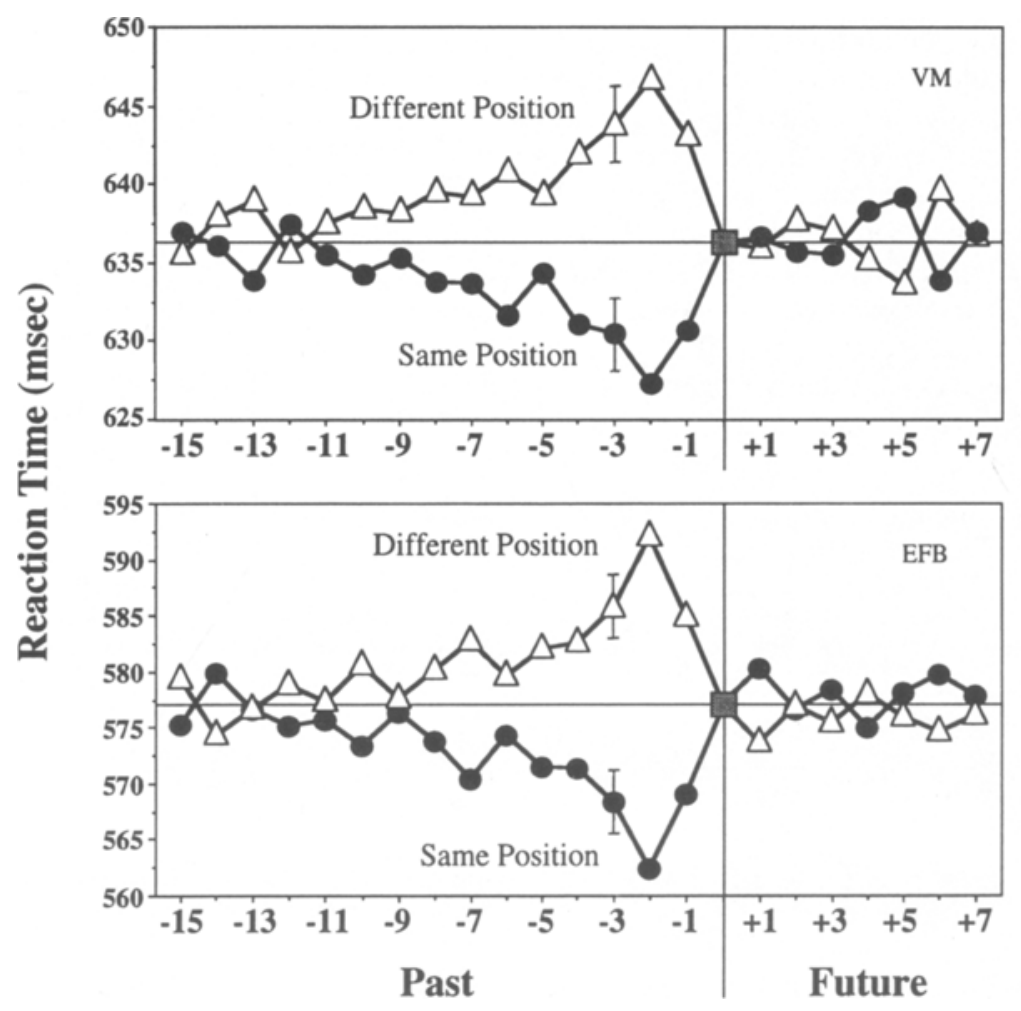

Figure 2. Reaction times for past trials in Experiment 1 in which the target fell in the same position as the target on the current trial (filled circles), and in which it fell in a different position (open triangles). Facilitation for the repeated position and inhibition for the changed position are greatest for trials just prior to the current trial. The effect falls off, but is still present for the trial five trials in the past. Note that these are effects of single trials in the past because the effects of the same and different positions of other trials are averaged out. 
ous trial, not the one that was one or two trials back. Error rates associated with position priming, though very low (1.32 $\pm 0.21 \%$ for V.M., and $3.23 \pm \mathbf{0 . 3 \%}$ for E.F.B. $)$, suggest that there is no speed-accuracy tradeoff. Moreover, if data from separate sessions are analyzed, the same pattern is observed repeatedly. There remains, therefore, a possibility that hidden in the data is subtle evidence for "inhibition of return" to the same position. It could only be a weak inhibition, however, as it clearly does not alter our major finding of an overall facilitation for the repeated position. Its effects would also have to be very short in duration. In that case the summing of facilitation with a very brief (say, even just one trial) inhibition of return would produce the results we obtained here.

\section{EXPERIMENT 2 \\ Spatial Properties of Position Priming: 6- and 12-Position Cases}

The previous experiment was well suited to examining the time course of position priming. However, as the position of the target in the two-position case was severely constrained (it could only stay in the same position or exchange positions with one distractor), we could learn little about the spatial extent of position priming. In Experiment 2, therefore, we varied the location of the target and distractors over a wider range of positions. Each target could assume one of 6 positions (for Observer V.M.) or one of 12 positions (for Observers G.P., G.A.O., and E.F.B.) on an imaginary clock face (the 12-position case is shown in Figure 3). This experiment, like the previous one, also allowed us to examine position effects over time. The question of the duration of the priming in cases of multiple positions is interesting for two reasons. First, it is possible that a single trial several trials in the past influences the response to the current trial in a complex pattern; for example, we can ask whether priming is restricted to just the position of previous targets or whether

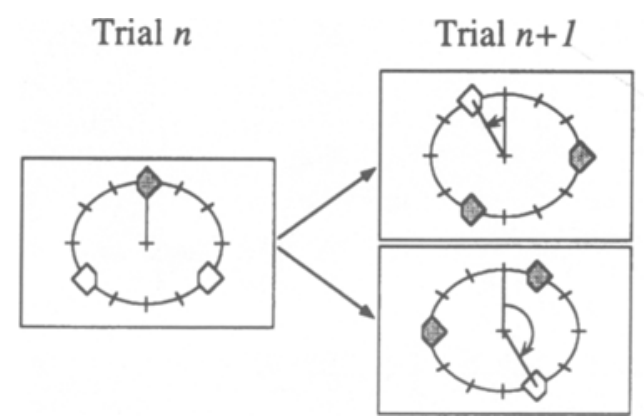

Figure 3. Diagram of positions examined in Experiment 2. The target could fall on any of the 12 positions shown. The distractors were equidistant from the target and from each other, so that, in effect, the whole display rotated around the ellipse. The data were analyzed in terms of distance between the target on Trials $n$ and $n+1$. That distance could be from zero, if the target stayed in the same position, to 6, if it moved to the opposite position on the ellipse. We show the cases of distances of 1 and 5 (top and bottom panel on the right, respectively). it spreads to neighboring positions. Second, the duration of position priming we observed (five to seven trials) may be unrepresentative of position priming in general. Using only two positions may have caused saturation, possibly increasing proactive inhibition, a phenomenon that often occurs in memory studies when trials are repeated. If this were the case, we might find that a more prolonged duration of priming would occur with more target positions.

In this second experiment we also used an additional variant of the paradigm - -judging the vernier offset of an odd spatial frequency Gabor patch, in which facilitation is observed with repetition of the spatial frequency (Maljkovic \& Nakayama, 1994).

\section{Method}

Subjects. Four observers participated in this experiment. Observer G.P. participated in the spatial-frequency task, while Observers E.F.B., V.M., and G.A.O. participated in three, slightly different, versions of the color task.

Stimuli. The stimuli for the spatial frequency experiment were Gabor patches, spatially localized sine-wave gratings whose modulation envelope is a 2-dimensional Gaussian function. Their suprathreshold area subtended approximately $1.8^{\circ}$. They were of either high ( 3.15 cycles/degree) or low ( 1.66 cycles/degree) spatial frequency, with the vernier offset of the top half of approximately $0.09^{\circ}$, to either the right or the left. The observer was required to respond to the direction of offset. The color stimuli for Observers E.F.B. and V.M. were the same as those described in Experiment 1 (red and green diamonds for E.F.B., and ocher-yellow and orange-yellow diamonds for V.M.). For all the observers, the color/spatial frequency of target and distractors switched unpredictably from trial to trial.

Observer G.A.O.'s data came from a different set of experiments primarily intended to understand interactions of multiple color pairs (to be described elsewhere). A stimulus was composed of either a $\mathrm{red} / \mathrm{green}$ or a blue/yellow combination of diamonds. The luminance of colors was chosen so that RTs for all stimuli were comparable. The luminance values were as follows: red, $1.65 \mathrm{~cd} / \mathrm{m}^{2} ;$ green, $1.11 \mathrm{~cd} / \mathrm{m}^{2}$; blue, $0.99 \mathrm{~cd} / \mathrm{m}^{2}$; and yellow, $1.91 \mathrm{~cd} / \mathrm{m}^{2}$. The CIE coordinates for the stimuli were as follows: red, .613/.344; green, $.325 / .571$; blue, $.150 / 062$; and yellow, $.481 / .448$. For the present purposes of examining position priming, this apparent increase in complexity did not alter the task from the observer's point of view - G.A.O. always responded to the shape of the odd-colored target.

The position of the target was chosen randomly for each trial for all 4 observers. The target and two distractors were positioned on the same, almost circular, ellipse described in Experiment 1, except that this time the target could fall on any of the 12 clock positions for Observers G.P., E.F.B., and G.A.O., and 6 positions $(2,4,6,8$, 10 , and 120 'clock) for Observer V.M. Distractors were always positioned so as to be equidistant from the target and each other, so that, in effect, the whole display rotated around the ellipse.

Procedure. The procedure was the same as that in Experiment 1 , the task being to indicate which side of the odd-colored diamond was cut off, or to which side the top of the odd spatial frequency patch was shifted. Observer G.P. ran 5,600 trials in seven sessions, Observer E.F.B. ran 4,000 trials in three sessions, Observer V.M. ran 4,400 trials in four sessions, and Observer G.A.O. ran 4,000 trials in four sessions. Trials were generally run in blocks of 200 , except for G.A.O.'s first two sessions, in which she ran 100 trials per block.

\section{Results and Discussion}

In Figure 4 we plotted the effects of the previous position on the current trial, then the duration of the position priming in Figures 5, 7, and 8. In Figures 4 and 5, we 

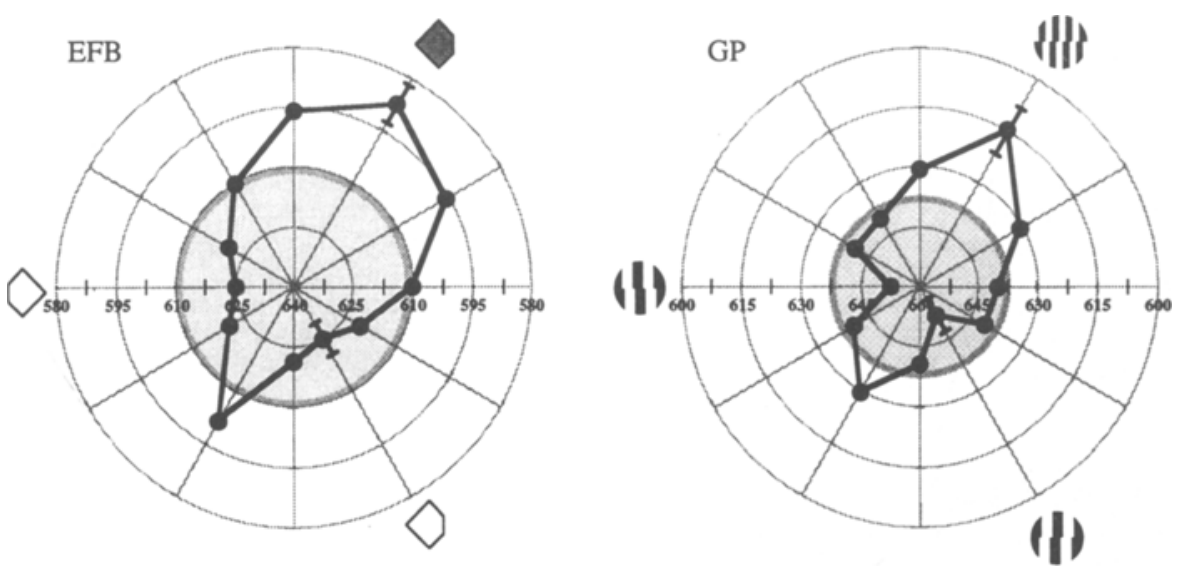

Figure 4. Polar plot of the effect of the preceding trial on the current trial in the color version (left) and the spatial-frequency version of the task (right) in Experiment 2. The independent measure is the distance between the target position on the preceding trial and the current trial. Long reaction times are plotted close to the inside and short reaction times close to the outside of the circle. The fastest responding occurred when the target position was repeated (the point underneath the target), and the slowest when the target fell where a distractor used to be (points next to distractor symbols). The bounds of the gray circle represent the overall mean reaction time.

plotted the data in polar coordinates, with positions normalized. Although in the experiment the whole display rotated unpredictably, in this plot we chose 1 o'clock to represent the current target position, then analyzed the preceding trial as if it had fallen in that same position or in any other position around the clock, relative to the target position.

We grouped the data according to the distance from the target position. For example, it can be seen in Figure 3 that the target on Trial $n+1$ moved one position away (shown in the upper panel) and five positions away (shown in the lower panel) compared to the target in Trial $n$. RTs for points the same distance from the target on either side were combined into a single value, which we plotted twice in order to show priming over the whole range. The distance of 0 , that is, when the target fell in the same position twice in a row, and the distance of 6 , when the target appeared at the location directly opposite the target, occurred on average half as many times as the others. We plotted standard error bars for both the target position (which is also an indicator of the standard error for the position opposite the target) and another standard error bar for the distractor position, as an indicator of the standard error for all other positions. The longer RTs are in the center of the plot, and the shorter RTs are on the outside. Thus, the closer to the outside a point falls, the faster the observer was for that position. The bounds of the grayshaded area represent the overall mean.

Figure 4 shows that when the target falls in the same position on two consecutive trials there is marked facilitation in both the color and the spatial frequency version of the task. Facilitation also occurs for the two positions adjacent to the target position, but it is smaller. When the target on the current trial falls in a location where a distractor appeared in the previous trial, there is inhibition. Smaller inhibition also exists for the position adjacent to the distractor position. The data therefore confirm the priming of target position and, in addition, reveal a separate negative influence of the distractor position. They also demonstrate that position priming is not confined to the target or distractor position but is spatially graded.

Next we address the question of the duration of this characteristic spatial signature of position priming. In Experiment 1 we saw that facilitation for the repeated target position and inhibition for the changed position lasted for five to eight trials. Would the pattern of results be different if there were more positions, so that the same two positions were not always tapped?

We performed the single-trial analysis for trials in the past using the same procedure as that in Experiment 1. Figure 5 shows the influence of past trials for Observer G.P. The complex pattern of facilitation and inhibition distributed around target and distractor positions, respectively, remains apparent even for the fifth trial in the past. This means that the position of the stimulus five trials in the past by itself influences response speed on the current trial. Moreover, it does so in more or less the same characteristic pattern as it does when it is the trial immediately preceding the current trial (see Figure 4).

At first it may appear quite surprising that a single trial in the past, roughly $20 \mathrm{sec}$ before, affects our deployment of attention in such a complex pattern. We therefore wanted to explore the duration of this position facilitation and inhibition more systematically.

We mentioned earlier that the target position could fall on any one of 6 or 12 positions around the ellipse from one trial to the next. We grouped positions from the 12 position case into categories, explained in Figure 6, and referred to these categories in Figures 7 and 8.

The target and distractors in Figure 6 are shown as they appeared on Trial $n-i$. On Trial $n$ (the current trial), 


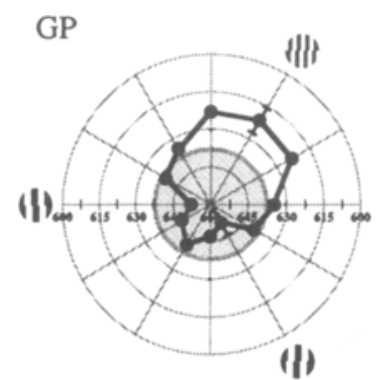

n-5

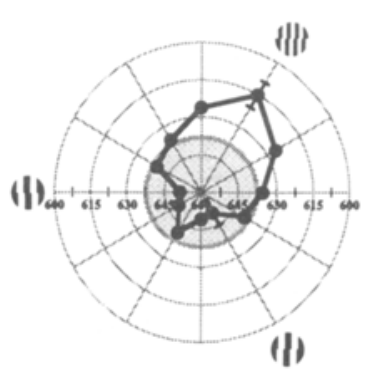

n-4

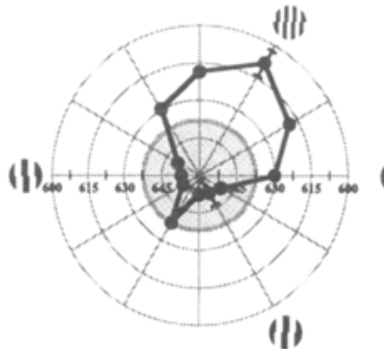

n-3

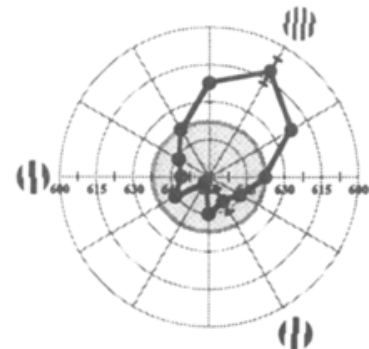

n-2

Figure 5. The effect of a single trial in the past on current target position in Experiment 2. The influence of the Trial $n-1$ for Observer G.P. is shown in Figure 4. Note that the same characteristic pattern of position priming is also present for the second through the fifth trial in the past.

the target is at the 10 'clock position, marked by the arrow. There are 6 categories of stimulus position that relate Trial $n-i$ and Trial $n$ (a single example is shown for each category): (1) The target stayed in the same position, marked "target"; (2) the target moved to the positions previously occupied by distractors, marked "distractor" (the whole display is rotating; the target is now where a distractor used to be); (3) the target moved to one of the two positions previously adjacent to it ("target-adjacent"); (4) the target moved to one of the four positions previously adjacent to distractors ("distractor-adjacent"); (5) the target moved to the opposite position ("opposite"); and (6) the target moved to one of the two remaining positions, marked "other," which are neither adjacent to the target or distractors nor opposite to the target. In Figures 7 and 8 we plotted on the $x$-axis the Trial $i$ in the past (and in the future, for a baseline), and on the $y$-axis we plotted the means of correct RTs for different conditions. Figure 7 presents the data for 6 positions for Observer V.M., and Figure 8 shows the results for the 12-position case for G.P. and G.A.O.
First let us consider the results for the 6-position case in Figure 7, where fewer locations could be explored. Because adjacent positions are always simultaneously adjacent to both the target and the distractors, these categories are absent.

The top panel shows what happens when the target on the current trial falls in the same position it did in the past (filled circles) with what happens when it falls on a position previously occupied by a distractor (open triangles). The bottom panel shows the data for the remaining two cases: the position opposite the target (open diamonds) and "other"; that is, positions adjacent to both target and distractors (filled squares). The target position shows clear facilitation for seven trials in the past, and the distractor position shows inhibition that appears to be somewhat shorter lived. We note here (and later) that target-position facilitation is greater than distractor-position inhibition.

In Figure 8 we show the data for the 12-position case. The left columns plot the data for Observer G.P., who ran the spatial-frequency version of the experiment, and the right columns present the results for Observer G.A.O.,

\section{Trial $n-i$}
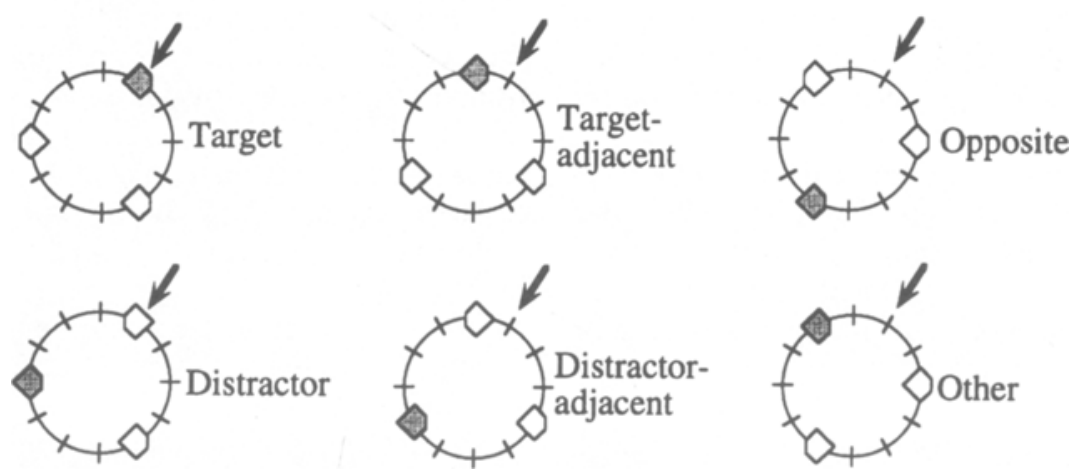

Figure 6. Definition of the categories of target positions used in Figures 7 and 8. Target and distractors are presented as they appeared on Trial $n-i$. The arrow marks target's position on Trial $n$. Left-most diagrams show cases in which the target either stayed in the same position or moved to a position previously occupied by a distractor. Middle diagrams show a position adjacent to the target (top) and one adjacent to a distractor (bottom); the right-most diagrams show the remaining possibilities. (Some categories contain additional possibilities; see text.) 


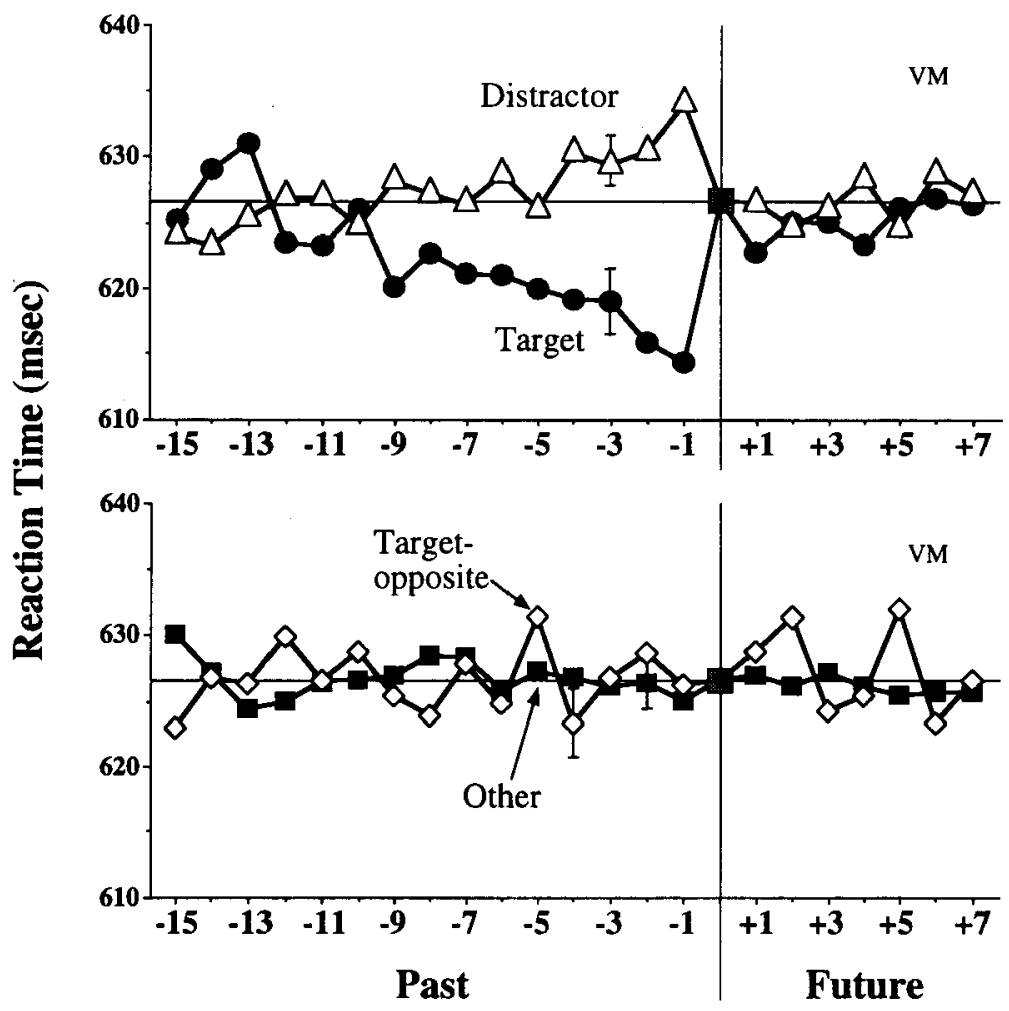

Figure 7. The effect of different positions in the past on the current target position for the six-position case (Observer V.M.) in Experiment 2. The top panel shows the influence of the previously target-occupied (filled circles) and distractor-occupied (open triangles) positions. The bottom panel shows the remaining positions. Repeating the target position produced facilitation; placing it where a distractor had been produced inhibition. The target effect was stronger than was the distractor effect, and both persisted for several trials in the past. The remaining positions show no priming.

who ran the color version. The top graphs show the "target" and "distractor" conditions and the bottom ones the "target-opposite" and "other" conditions; we also examined the effect of positions adjacent to target and distractors, as presented in the middle row of graphs.

First, note the facilitation for the "target" position and the inhibition for the "distractor" positions. These effects are practically the same as those shown in the 6-position case: Facilitation is evident for at least seven trials in the past, and inhibition is of smaller magnitude and of somewhat shorter duration. Second, note the similar lack of priming for the "target-opposite" and "other" positions shown in the bottom row. Finally, consider the middle row of graphs, which details the influence of positions adjacent to either target (filled circles) or distractors (open triangles). These adjacent positions show a priming pattern similar to that of target and distractors - that is, target-adjacent positions are facilitated and distractoradjacent positions are inhibited. Facilitation is, again, greater than inhibition, but both are diminished compared with facilitaton and inhibition for actual target and distractor positions. Thus a single trial several trials in the past continues to influence responses for several subsequent trials in a very characteristic pattern.
Priming effects examined in memory research typically last for periods of time significantly longer than those reported here (e.g., Schacter, Chiu, \& Ochsner, 1993). Is this memory phenomenon qualitatively different from those reported in the previous accounts? Or, alternatively, is it due to the very different methods that we have used to probe for priming, specifically in the frequent repetition of a tiny set of potential memory items? Perhaps the relatively rapid decay of memory for position is a result of proactive and retroactive interference caused by the unusually large number of same-stimuli repetitions prior to or after the current stimulus (Baddeley, 1986). This would be especially true for the 2-position case. We thus hoped to address the question of interference through the multipleposition experiment. Because each position appears less frequently, there is less interference and memory may last longer. Our results do not show a clear increase in the duration of priming for the multiple-position case. Thus, over a fairly large range of conditions-from those in which previous target and distractors are frequently repeated (as in Figure 2), to cases in which such repetitions are less frequent (see Figure 8)-there is no obvious difference in the time course of priming. Memory for position appears to indeed be relatively short-lasting. 

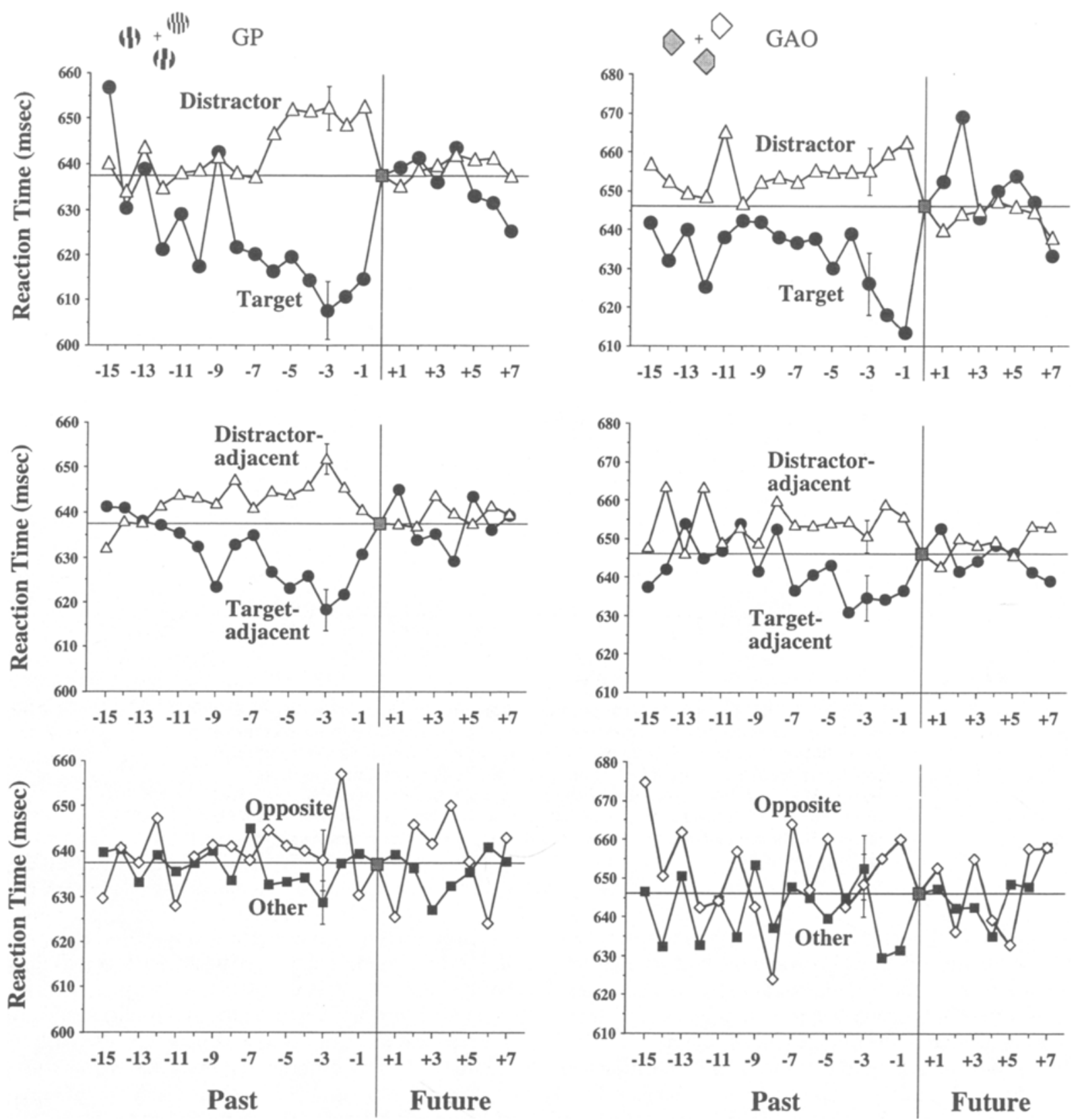

Figure 8. Effect of the different positions exerted by a single trial in the past on the current trial in Experiment 2. The top row shows the influence by target and distractor positions; the middle row shows influence by target-adjacent and distractor-adjacent positions; and the bottom row shows the data for the remaining positions. Note that facilitation and inhibition for the "target" and "distractor" positions, and absence of priming for the "opposite" and "other" conditions, is analogous to the finding for the sixposition condition. In addition, facilitation and inhibition effects (somewhat diminished) are present for "target-adjacent" and "distractor-adjacent" positions; and, again, facilitation is stronger than inhibition and diminishes over time.

These results amply confirm and extend the results of our first experiment. Position priming has a very characteristic signature, both in space and time. Attentional deployment is facilitated for a target landing on a past target position, whereas it is inhibited for a target landing on a past distractor position. Furthermore, the effects are graded over both time and space. Priming falls off gradually over time and also varies according to the relative proximity to an earlier target or distractor.

\section{Independence Versus Conjunction} of Feature and Position Priming

We showed in our first paper (Maljkovic \& Nakayama, 1994) that pop-out is primed by repetition of the attentiondriving feature and, in this paper, that it is also primed by repetition of the stimulus position. These two priming effects occur when the two variables change completely independently of each other, as they did in our experiments. Nevertheless, we need to look more carefully for 
a possible mutual interaction. Although the odd color and odd spatial frequency are critical for performing the task and are directly chosen by observers, target position is not. From the observer's point of view, therefore, position is an irrelevant variable. Earlier studies suggest that position is primed only when target color is primed. Treisman, Vieira, and Hayes (1992) and Park and Kanwisher (1994) found that position-priming effects exist when the associated feature shows priming. Tipper et al. (1990) found that "negative priming goes with the object," that is, when the object to be ignored moves to a new location, negative priming occurs relative to it. J. Miller (1988), who found location-based facilitation to be partly target dependent and partly target independent, showed that the target-dependent effect is stronger. These findings are in line with the suggestion of Treisman (1988) that objects are the units coded, and, therefore, that effects of other attributes are tied to the objects.

Do we have any evidence for object-specific priming in our task? In other words, is there evidence for priming that is based on an assocration between a feature (say, color or spatial frequency) and its previous position? We address this question by analyzing the data summarized in Figure 4 in more detail. In Figure 9 we separately plotted RTs for the previous position when the preceding trial feature was the same and when it switched. If priming is confined to the "object," we would expect that when the color switched the characteristic pattern of priming would either disappear or become greatly attenuated. More specifically, we would expect the pattern of RTs to become circular for the "preceding-color-different" case. Contrary to this expectation, the data shown in Figure 9 indicate that position priming is comparable, even when the feature switches.

Same-feature RTs for all preceding positions were faster (closer to the outside of the circle) than were differentfeature RTs (closer to the center of the circle). The general shape of position priming, however, is the same for the two cases: there is facilitation for the repeated posi- tion and inhibition for the distractor position regardless of whether the previous feature was the same or different. There is no hint that the inner plot becomes more circular. The results suggest, therefore, that position priming is essentially independent of feature priming.

\section{EXPERIMENT 3 \\ Spatial Coordinate Representation for Position Priming}

Finally, we examined the spatial coordinate frame in which position priming takes place. Both negative priming (Tipper et al., 1990) and inhibition of return (Maylor, 1985; Tipper, Driver, \& Weaver, 1991) have been shown to be coded in object-centered coordinates. J. Miller (1988) found that location-based facilitation was partly predicted by absolute spatial coordinates and partly by the position of the target within the display. The question of position priming is interesting both in terms of the level of representation that is primed and, potentially, the brain area that subserves the effect.

In our companion paper on feature priming, we presented two findings relevant to this question. Color priming showed complete binocular transfer, suggesting that it takes place after the binocular combination of neural signals. We also found that presenting the display in the same hemifield on successive trials conveyed no benefit compared with presenting it in different hemifields. Both sets of results indicate that feature priming occurs fairly late in visual processing and is not coded retinotopically. They do not bear, however, on the question of whether position priming is retinotopic or object centered.

To answer this question, we employed a somewhat different display from the one used in previous experiments. It was designed to dissociate absolute and relative positions. Instead of having the target and distractors rotate around an ellipse, we used a single horizontal row of three elements (Figure 10), in which the target could be in the left, middle, or right position. The horizontal row appeared

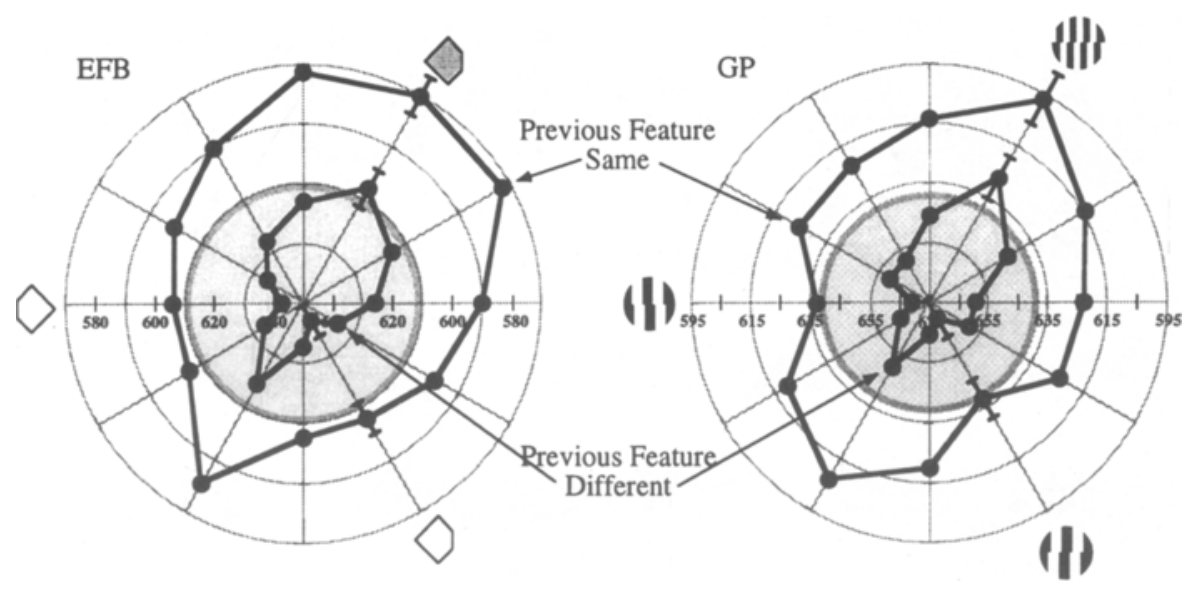

Figure 9. Independence of position and feature priming. When the preceding trial had the same-feature target (outside circle), reaction times were shorter. When it was different they were slower, but the shape of the position priming was the same in both cases: The shortest reaction time was associated with the repeated target position and longest time with the distractor positions. 
in one of four absolute locations on the screen, and the target in one of three relative positions within the horizontal row. The display was devised by Mary Bravo, and initial results using the current version were reported earlier by Maljkovic, Bravo, and Nakayama (1992).

To measure the contribution of a landmark- or objectcentered component alone we took advantage of the cumulative nature of the priming - that is, the fact that with each successive repetition RT is further reduced (Maljkovic \& Nakayama, 1994). By isolating a particular feature and repeating it while changing all the others, we can observe how quickly priming accumulates over several trials. We can thus compare any two conditions by comparing their cumulative priming effects. In this experiment, we show how much priming is observed when both the absolute and the relative target positions are repeated, versus when only the relative position is repeated but the whole display is shifted around the screen. ${ }^{7}$

\section{Method}

Subjects. Three observers, authors K.N. and V.M., and a naive observer, F.T., participated in this experiment.

Stimuli. Elements were red and green diamonds for observers K.N. and F.T., and orange-yellow and ocher-yellow diamonds for observer V.M. (as described in Experiment 1). Each trial employed a single target of one color and two distractors of the other color. The elements were arranged in a single horizontal row that, together, subtended approximately $4.5^{\circ}$ of visual angle. The target could be in the left, center, or right relative position within the display; in addition, at each trial, the target (but not the distractors) was jiggled randomly from 0 to 5 pixels in a vertical direction. The target could fall at one of four fixed positions on the screen that marked the corners of a central square with sides $6.78^{\circ}$ of visual angle; a fixation point, as described in Experiment 1, was in the middle of the square. The target could always, therefore, be described in terms of both its absolute position on the screen (upper left, upper right, lower left, or lower right) and its relative position within the display (left, center, or right).

Procedure. We used short sequences of repeated position trials. Within each sequence, one of two conditions was enforced: (1) Both the absolute and the relative position of the target were the same from trial to trial; or (2) the relative position of the target was the

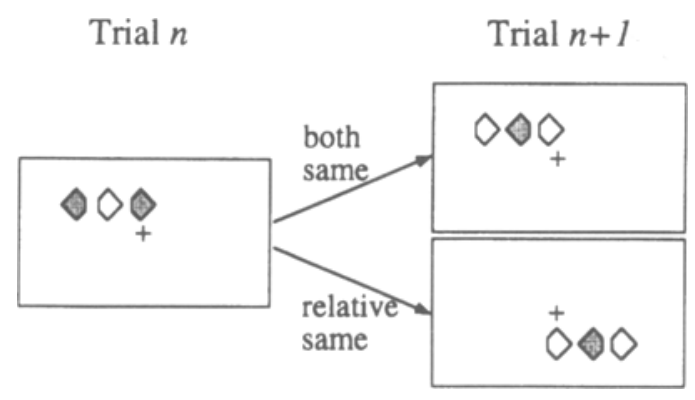

Figure 10. Schematic of the display viewed in Experiment 3. The absolute position of the target could be one of the four corners of an imaginary square around the fixation point; its relative position could be in the left, center, or right position within the display. From Trial $n$ to Trial $n+1$, the target either stayed in the same absolute and the same relative position ("both same"), or it stayed in the same relative position but moved to another absolute position ("relative same") on the screen. same but the absolute position changed with each trial (these conditions are represented in Figure 10).

In the first condition the whole display stayed in the same position over a short sequence; in the second case, while the whole display constantly changed position on the screen, the target within it always stayed in the same relative position. In between the trials of these conditions, a three-trial sequence in which both the absolute and the relative position changed on each trial was presented, to reset the priming for the beginning of the new test sequence. The new sequence always began with the relative and absolute positions of the target different from those in the trial just preceding it.

The sequences could be either three, six, or nine trials long for $\mathrm{Ob}$ server K.N., three or five trials long for Observer V.M., and three or six trials long for Observer F.T. The length of any sequence was unpredictable. In this experiment, the color was much more likely to change than to stay the same---the probability that the color would switch on the next trial was 0.85 , rather than the usual 0.5 .

The eye movements of K.N. were not monitored; as he is a practiced observer, he monitored his own eye movements by staring at the fixation point during the experiment. When, in this case, the eyes move, a trained observer can easily perceive an afterimage of the fixation point at the location to which the eyes move. K.N. reported making no more than one or two eye movements per block. We monitored the eye movements of Observers V.M. and F.T. by using an infrared sensing system (Ober2). The head of Observer V.M. was fixed by a bite bar, and Observer F.T. rested his head on a chinrest; the first four trials of each block were used for calibration. In each of the initial trials, the target appeared in the central position within the stimulus and the stimulus was shown at all four absolute locations, and the observer made a saccade to the target. For the rest of the block the observer fixated and attempted not to make any saccades toward the targets. This proved to be extremely easy for the author, V.M. (saccades were made in only about $1 \%$ of the trials), and considerably more difficult for the naive Observer F.T. (saccades were made in $22 \%$ of the trials). Trials in which an eye movement greater than $1^{\circ}$ occurred, as well as the subsequent trials within that sequence, were discarded from analysis. $\mathrm{Ob}$ server K.N. ran 2,400 trials in two sessions. Observer V.M. ran 1,200 trials in blocks of 30 to 100 trials over three sessions. Observer F.T. ran 550 trials in blocks of 100 to 200 trials in a single session.

\section{Results and Discussion}

The data are shown in Figure 11. The $x$-axis represents the order of the trial within a sequence - whether the target was presented first, second, third (etc.) in the same absolute and relative (solid circles) position or only in the same relative position (open triangles). The $y$-axis represents the RT recorded for a correct response.

We note first that repetitions in both conditions led to an improvement over the trials in the sequence for all 3 observers - another replication of the position priming effect demonstrated in this paper. This finding is also evidence that position priming is cumulative; that is, that the effects of single trials in the past summate with others to increase the priming effect. Second, data from all 3 observers show an overall similarity, suggesting that K.N.'s results are not due to unintentional eye movements. Finally, all observers showed a slight but consistently greater priming when both the absolute and relative target position remained the same than when only the relative position stayed constant. The object-centered or landmark-centered component thus seems to account for a sizable portion, although not all, of position priming. 

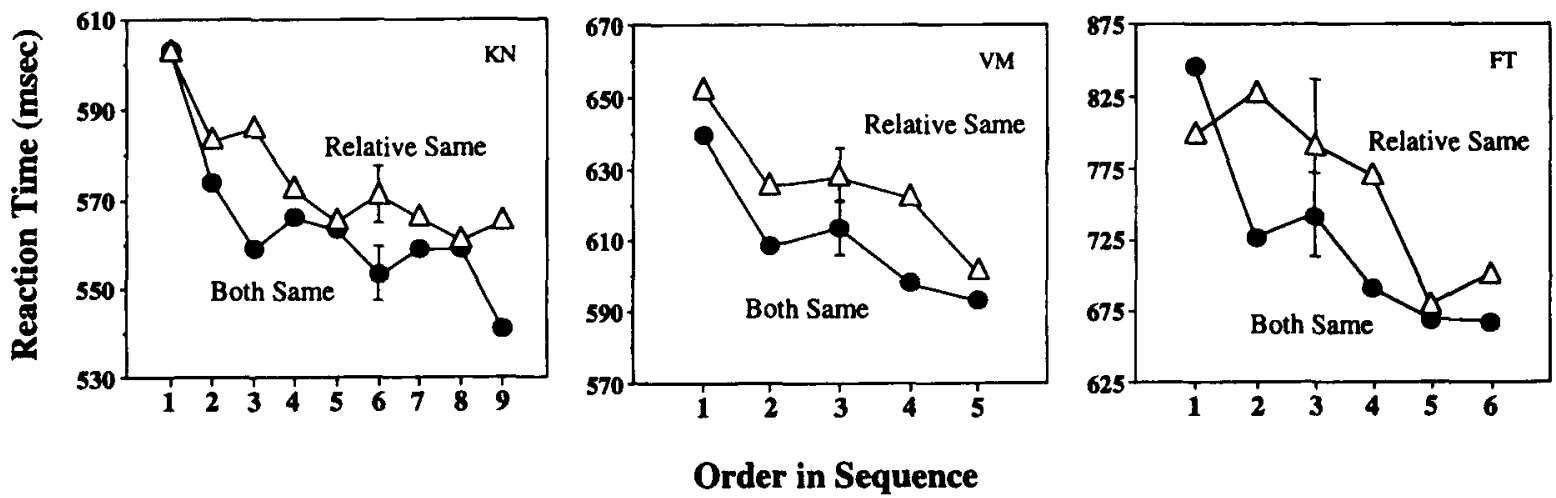

Figure 11. Shown is the cumulative effect produced when both the absolute and the relative position of the target repeated for a short sequence of trials ("both same," solid circles) and when only the relative position repeated from trial to trial ("relative same," open triangles) in Experiment 3. Note that when the relative position alone repeated, the effect of priming was similar to that found when both positions stayed the same, suggesting that position priming is largely object centered.

\section{GENERAL DISCUSSION}

Our earlier study indicated that deployment of attention to an odd "pop-out" target is dictated not only by the characteristics of the visual-stimulus display (see Bravo \& Nakayama, 1992), but also by very specific properties of past stimulation. In this paper, we have described one form of this memory phenomenon, position priming, showing that it is graded in both time and space. In the time dimension, the data demonstrate that a single exposure can leave a characteristic residue lasting approximately five to eight trials. The priming is cumulative, such that the effects of repeating a target in the same position summate, leading to a very substantial speeding up of attentional deployment. In the space dimension, the priming also has a characteristic and graded profile. Targets appearing at previously placed target positions show facilitation, while targets appearing at previously placed distractor positions show inhibition. Adjacent positions of previous targets and previous distractors also show facilitation and inhibition, respectively, but with reduced amplitude. Finally, the coordinate frame for position priming is not based on retinal loci but appears to be largely centered on objects or landmarks.

\section{Similarities Between Position and Feature Priming}

Position priming is very similar to the feature priming reported earlier (Maljkovic \& Nakayama, 1994). The two types of priming share at least four characteristics: (1) Each can be either facilitatory or inhibitory; (2) their time course is approximately the same, lasting for five to eight trials; (3) they have approximately the same magnitude, the maximum size of the priming from a single trial being in the range of 20 to $50 \mathrm{msec}^{;}{ }^{8}$ and (4) each type can show summation - that is, priming from successive same-color or same-position trials is cumulative and results in large decreases in RT.

A comparison of position and feature priming indicates that these very robust effects are mediated by many separate yet similar processes: target-feature facilitation and distractor-feature inhibition (described in Maljkovic \& Nakayama, 1994) and target-position facilitation and distractor-position inhibition (reported here). Each process is graded and, as a result, various positions or features are either positively or negatively valenced for purposes of attentional deployment. Each of these multiple tendencies appears to coexist, essentially independently of the others. This is best illustrated in Figure 9, which shows examples of position priming in cases in which the target feature remained the same and in cases in which the target feature changed from the previous trial. Feature priming is reflected in the fact that the same-feature target function (outer curve) is faster than the different-feature target function (inner curve). Position priming is also indicated by the preserved shape of the two polar plots, each of which shows target-position facilitation and distractorposition inhibition.

Furthermore, preliminary evidence suggests that popout priming is likely to fall into the category of short-term memory phenomena. It appears not to be a long-term memory subject to strong proactive or retroactive inhibition; adding a greater number of positions, as in Experiment 2 , does not prolong the priming, as would be expected for a long-term memory.

In a later discussion, we document more exhaustively just how different priming of pop-out is in relation to priming reported by other researchers. This priming is sufficiently novel to dwell on what we see as its defining characteristics, and to draw a physical analogy underscoring those essential features.

\section{A Physical Analogy}

Clearly, one of the most striking characteristics of this type of priming is the relatively fast decay of the memory process and the fact that it appears to summate. Thus sequences of same-position or same-feature trials have a cumulative influence. The underlying hypothetical process is illustrated in Figure 12. In panel A, we show the priming resulting from a single trial and its decay. In panel B, 
we show how priming from multiple trials accumulates so that priming lasts for more than a single trial.

Such processes of decay and summation are seen in many physical systems. Heat, for example, decays over time, and multiple increases of heat to a body summate and also decay (Newton's law of cooling). A more graphic comparison (allowing for positive and negative influences) is the simplest of electronic circuit elements-a parallel resistive-capacitative configuration-depicted in panel $\mathrm{C}$ of Figure 12. As each charge added is added to the input, the storage in the capacitor increases, the duration of the increase being dependent on the capacitance (C) and the resistance (R) through which it is discharged.

We speculate that the priming seen for position and features is mediated by a process roughly analogous to this physical model. Yet our work suggests that multiple and essentially independent elements, such as those depicted in panel $\mathrm{C}$ of Figure 12, are needed to account for the full range of our data. The present experiments indicate positive priming for positions previously occupied by a target but negative priming for positions occupied by a distractor. Similarly, we see a corresponding positive and negative priming for target and distractor features of color and spatial frequency (Maljkovic \& Nakayama, 1994). These findings lead us to propose that the salience of many different properties of a stimulus (different features and different positions) are all stored separately (Figure 13), and that the valence for each, either positive or negative, is incremented and decremented independently. The attractiveness of a given target in relation to others, therefore, depends on the sum of the priming "charge" of each of the elements associated with the position and features of the target.

\section{Function for the Priming of Attentional Deployment}

In this and in our earlier companion paper, we characterized a marked increase in performance in cases where position and/or features stay the same over multiple trials. Our finding that RTs can be much shorter for cases in which same features or positions are repeated raises the question of the functional value of priming. How might priming help in the deployment of attention in everyday life?

An important and obvious conclusion emerges from the work we have done so far: The position and feature priming in our experiments serve no functional purpose. Because the sequences of colors and positions are generally chosen at random, priming bestows no advantage. Yet in spite of this lack of functional benefit, in our experiments, short-term priming exists, even after the subject has run tens of thousands of trials. ${ }^{9}$ The durability of priming in the absence of any functional advantage in our experiments strongly suggests the existence of a robust mechanism that must be advantageous elsewhere.

Thinking along these lines makes it obvious that priming would be most beneficial in situations in which the focus of attention must be directed efficiently to temporary repetitions occurring over seconds or minutes. It would not be particularly relevant for shorter periodssay, a few hundred milliseconds-nor for longer periods of, say, hours or days.

One area in which such priming could be very useful is when complex sequences of action are repeated and attention to a specific type of item or a specific position is needed to serve some intermediate goal. For example, consider the repetitive motor activities involved in food preparation-for example, reaching for carrots, cutting them up, and throwing them into a pot. Here the automatic color priming for orange resulting from several attentional deployments would help redirect attention to the next uncut item. When switching to another class of vegetables, however, the short duration of color priming would also help us adapt quickly to the new task. Thus, the simple rough-and-ready nature of priming we have observed could aid efficient deployment of attention without requiring a sophisticated higher-order visual representation.

In animal behavior, ethologists have postulated the existence of a search image used in foraging and prey

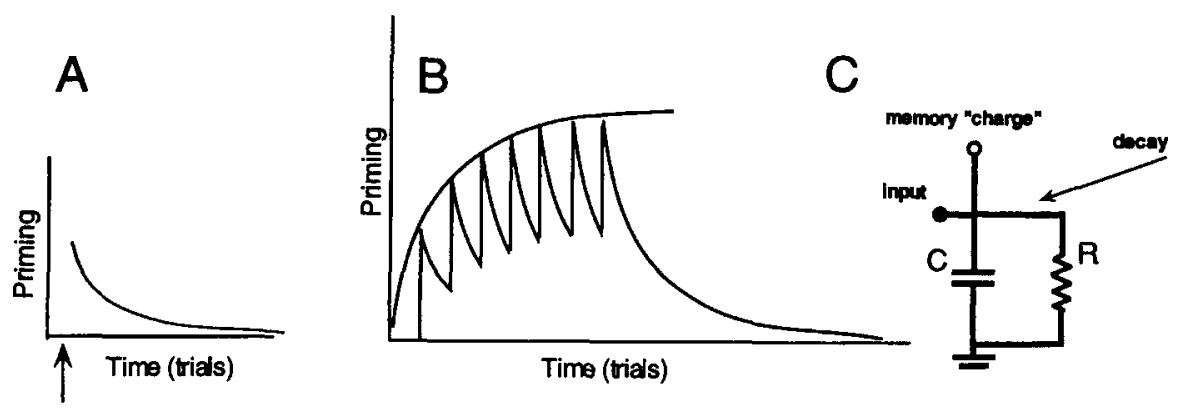

Figure 12. (A) Idealized description of the priming effects of a single trial. An attention-driving target characteristic is presented at the arrow leading to priming, which decays over time. (B) IIlustration of how the characteristics of such priming could accumulate over trials, assuming additive superposition of individual priming effects. (C) A parallel resistive-capacitative (RC) element that mimics such cumulative yet decaying effects. The element is charged by inputs because of the capacitor $C$; yet it also decays because of the shunting resistor $R$. The "memory charge" determines the attractiveness or salience in summoning attention (both positive and negative). 


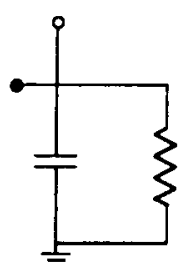

feature 1

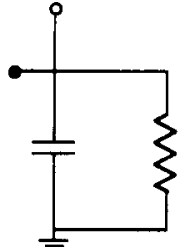

feature 2

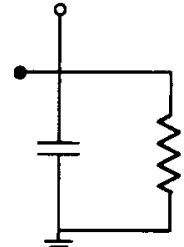

position 1

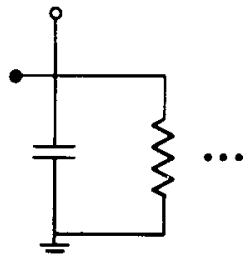

position 2

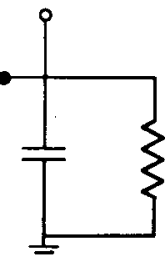

position $n$

Figure 13. Multiple memory elements, each associated with a different stimulus attribute (feature and position). The "memory charge" on each of these hypothetical elements determines the attractiveness (both positive and negative) of that particular stimulus attribute in terms of its attentionsummoning power. The net attractiveness of a given target is the resulting sum of the charge on the associated position and feature elements.

capture. It has been reported, for example, that when an animal finds food of a particular color, it continues to approach the same-color food, even when food of other colors is also available. Once successful for a particular color, the animal appears to orient itself solely to recently attended colors (Reid \& Shettleworth, 1992). Bees, too, on leaving the hive in the morning, define the color sought that day by the first flower they encounter that yields nectar (Menzel, 1987). This search image may have underpinnings similar to those of the priming we have described for the human visual system.

These speculations are, of course, suggestive only, and we acknowledge that the analogy to everyday life is incomplete. In the priming of pop-out, we consider the deployment of attention in a situation in which one sees an odd target flashed among others. For the deployment of attention in everyday life, the stimulus situation is likely to be more heterogeneous and the intentions and goals of the subject less specified. Still unresolved, therefore, is the issue of whether the priming of pop-out reflects the characteristics of attentional deployment in general or whether it is restricted to situations like those described, in which attention appears to be deployed with little effort or intention.

\section{Mechanism and Locus for Priming}

Our results indicate that the mechanism underlying pop-out priming, both for position and for color, is very primitive. Much of it can be accounted for by the simple storage of valence, as outlined in Figures 12 and 13. This finding is to be distinguished from the results of other priming studies (Park \& Kanwisher, 1994; Tipper et al., 1990) that show that specific objects are primed, not their features or positions separately - that is, that a target appearing in the same location would not show priming if it had changed its color. The characteristics of the priming of pop-out are thus different and more primitive. Despite the lack of a representation of specific objects, it should be clear that such priming could be useful in a variety of situations, both when features and positions co-occur with regularity and when they do not.

It is of considerable interest that position priming is not based on retinotopic coordinates but on a coordinate system defined by objects or landmarks in the world. This would make it particularly adaptive in normal real-life situations (as opposed to the laboratory) in which landmarks assume different retinal positions during body, head, and eye movements. Such an object-centered or landmark-centered view of vision and visual attention has received considerable emphasis in recent years. A number of researchers, primarily those interested in robotic vision (Bajcsy, 1988; Ballard, 1989) have postulated the necessity of world-centered representations for motor control. We suggest that the short-term memory we discovered may underlie such a representation and that its function may be to reorient our gaze more efficiently. The finding that saccadic latencies are reduced to repeated same-color targets and same positions (McPeek \& Nakayama, 1995; Skavenski, McPeek, Maljkovic, \& Nakayama, 1993) reinforces this hypothesis.

If the gaze-reorientation hypothesis is true, a possible neural locus may be the posterior parietal cortex. This area is involved in the spatial coding and transformations necessary for correct eye positioning (Andersen, 1987; Andersen \& Zipser, 1988; Goodale, Milner, Jakobson, \& Carey, 1991). Even if the hypothesis is not true, priming must happen fairly late in the stage of visual information processing because, in Experiment 3 (Figure 11), we showed that substantial amounts of priming are object or landmark centered. This is consistent with our earlier findings on color priming (Maljkovic \& Nakayama, 1994), in which we showed that such priming completely transfers from one eye to the other, as well as from one hemifield to the other. All these findings suggest that priming of attentional deployment must occur at a level at which retinotopy is not preserved and at which the coordinate framework is more abstract. These considerations would seem to rule out the involvement of striate cortex or adjacent extrastriate cortex, where retinotopy is still apparent. This leaves us with higher cortical visual areas. If we consider the possibility that priming takes place in the inferior temporal cortex (IT), we are drawn to the study of E. K. Miller, Li, and Desimone (1991). They found that in rhesus monkeys there is a decrease in firing in IT neurons with chance repetition of pictures.

Physiological evidence on this point is absent. We suggest that the subject of priming of attentional deployment is a suitable candidate for a physiological and/or ana- 
tomical analysis. Its presumed high-level visual representation has a strikingly machine-like, passive nature. It not only follows very simple primitive rules, but it also seems impervious to various changes in behavioral state or various expectancies. For example, in Maljkovic and Nakayama (1994), we showed that priming could not be overcome, even in highly predictable sequences in which observers knew the trial color on every upcoming trial. The study of the neural locus of this priming is made easier not only by its full characterization, but also by its relatively small dependence on fluctuations of the behavioral state.

\section{Relation to Other Position-Priming Studies: Inhibition of Return}

A number of recent studies suggest that repeating a target position on subsequent trials can alter performance. Before reviewing these studies, however, we should note that most of them are very different from the present series of experiments. The reader will recall that in our experiments we have taken considerable pains to ensure two things. First, we used a task requiring focal attentionthe shape differences used (cut-off diamond, vernier acuity) being too subtle to discriminate without focusing attention on the target site. Second, we dissociated the feature that drives attention from the feature that requires the observer's response. This enabled us to examine the priming of attentional deployment, not, for example, the priming of pattern recognition or object recognition. What is primed is not, therefore, the object the observer had to discriminate.

To compare our work to previous studies on priming, we first turn to situations in which targets are presented in the same position in consecutive trials. Two types of results are apparent. The most frequently reported finding, which seems to contradict the results of the present study, is a worsening of performance for targets that repeatedly appear in the same location (Kwak \& Egeth, 1992; Maylor \& Hockey, 1985; Posner et al., 1984). Such a phenomenon was dubbed "inhibition of return." These researchers concluded that visual attention had a tendency not to return to the same location twice. The notion was theoretically attractive because it seemed to provide a mechanism for understanding prevalent conceptions of visual search, in particular, the widely accepted view that in serial visual search, search slopes per item were twice the size for the target-absent than for the target-present case. Inhibition of return would allow the serial search to occur without retracing the attention to already scanned items. Examined in greater detail, however, inhibition of return is an inadequate explanation for the task, as it requires that the inhibition last for more than one trial. More recent models for explaining serial search have not, therefore, relied on it (Wolfe, Cave, \& Franzel, 1989).

To distinguish these results from those reported in the present paper, we should note that all the studies under the rubric of "inhibition of return" were simple detection tasks requiring the subject to indicate the presence of a target. Nakayama and Mackeben (1989), following Shaw
(1984), showed that such classes of simple detection do not require much in the way of focal attention, particularly when compared with the type of focal attention needed for a localized discrimination. Our interpretation is that the studies showing a slight disadvantage for repeated target position were not designed to require that focal attention be allocated to a localized position in the visual field. They probably constitute a very different type of finding unrelated to the deployment of focal attention. As such, it is possible that position priming of the sort we characterize in this paper occurs only in situations where attention must be rapidly deployed to a new site. It is possible, therefore, that both inhibition of return and position priming can occur at the same time but that position priming overshadows inhibition of return when it is present.

Several additional pieces of evidence are consistent with this view. The first comes from a study by Tanaka and Shimojo (1993), who replicated inhibition of return by using detection of a target. Changing it so that it becomes a discrimination task (thus requiring focal attention) reversed the sign of the priming so that it was weakly positive. A second piece of evidence comes from study of smooth pursuit eye movements that require focal attention for their execution. Kowler et al. (1984) showed facilitation analogous to the position priming reported here for eye movements to repeated positions. The third confirmation, mentioned in our description of the results in Experiment 1, is the greater position priming effect of more remote trials, as compared with the effect of the immediately preceding trial. While this result could be construed as evidence for inhibition of return, it would seem to be a relatively weak phenomenon, transient and overshadowed by the strong position priming reported here.

\section{REFERENCES}

ANDERSEN, R. A. (1987). Inferior parietal lobule function in spatial perception and visuomotor integration. In F. Plum \& V. B. Mountcastle (Eds.), Handbook of physiology: Vol. 5. The nervous system (pp. 483518). Bethesda, MD: American Physiological Society.

ANDERSEN, R. A., \& ZiPSER, D. (1988). The role of posterior parietal cortex in coordinate transformations for visual-motor integration. Canadian Journal of Physiology \& Pharmacology, 66, 488-501

BADDElEY, A. (1986). Working memory. New York: Oxford University Press.

BaJCsy, R. (1988). Active perception. Proceedings of the IEEE, 76, 996-1005.

BALLARD, D. H. (1989). Behavioral constraints on animate vision. Image \& Vision Computing, 7, 3-9.

Bravo, M. J., \& Nakayama, K. (1992). The role of attention in different visual-search tasks. Perception \& Psychophysics, 51, 465-472.

Goodale, M. M., Milner, A. D., Jakobson, L. S., \& Carey, D. P. (1991). A neurological dissociation between perceiving objects and grasping them. Nature, 349, 154-156.

Kowler, E., Martins, A. J., \& Pavel, M. (1984). The effect of expectations on slow oculomotor control-IV. Anticipatory smooth eye movements depend on prior target movements. Vision Research, 24, 197-210.

Kröse, B. J. A., \& JulesZ, B. (1989). The control and speed of shifts of attention. Vision Research, 29, 1607-1619.

KWAK, H.-W., \& EgETH, H. (1992). Consequences of allocating attention to locations and to other attributes. Perception \& Psychophysics, $51,455-464$. 
Maljkovic, V., Bravo, M., \& Nakayama, K. (1992). Characteristics of short-term visual memory which passively influence the guidance of focal attention. Perception, 21 (Suppl. 2), 68.

Maljkovic, V., \& Nakayama, K. (1994). Priming of pop-out: I. Role of features. Memory \& Cognition, 22, 657-672.

MAYLOR, E. A. (1985). Facilitatory and inhibitory components of orienting in visual space. In M. I. Posner \& O. S. M. Marin (Eds.), Attention and performance $X I$ (pp. 189-207). Hillsdale, NJ: Erlbaum.

MAYLOR, E. A., \& HOCKEY, R. J. (1985). Inhibitory component of externally controlled covert orienting in visual space. Journal of Experimental Psychology: Human Perception \& Performance, 11, 777-787.

McPeek, R. M., \& Nakayama, K. (1995). Linkage of attention and saccades in a visual search task. Investigative Ophthalmology \& Visual Science, 36(4), S354.

MenZel, R. (1987). Memory traces in honeybees. In R. Menzel \& A. Mercer (Eds.), Neurobiology and behavior of honeybees (pp. 310325). Berlin: Springer-Verlag.

Miller, E. K., LI, L., \& Desimone, R. (1991). A neural mechanism for working recognition memory in inferior temporal cortex. Science, 254, 1377-1379.

MILLER, J. (1988). Components of location probability effect in visual search tasks. Journal of Experimental Psychology: Human Perception \& Performance, 14, 453-471.

Nakayama, K., \& MaCkeben, M. (1989). Sustained and transient components of focal visual attention. Vision Research, 29, 1631-1647.

Nejll, W. T., Terry, K. M., \& Valdes, L. A. (1994). Negative priming without probe selection. Psychonomic Bulletin \& Review, 1, 119-121.

Park, J., \& Kanwisher, N. (1994). Negative priming for spatial locations: Identity mismatching, not distractor inhibition. Journal of Experimental Psychology: Human Perception \& Performance, 20, 613623.

Posner, M. I., Cohen, Y., Choate, L. S., Hockey, R., \& Maylor, E. (1984). Sustained concentration: Passive filtering or active orienting? In S. Kornblum \& J. Requin (Eds.), Preparatory states and processes (pp. 49-65). Hillsdale, NJ: Erlbaum.

Reid, P. J., \& Shettleworth, S. J. (1992). Detection of cryptic prey: Search image or search rate? Journal of Experimental Psychology: Animal Behavior Processes, 18, 273-286.

Rovamo, J., \& Virsu, V. (1979). Visual resolution, contrast sensitivity, and the cortical magnification factor. Experimental Brain Research, 37, 475-494

Schacter, D. L., ChiU, C.-Y. P., \& OChSner, K. N. (1993). Implicit memory: A selective review. Annual Review of Neuroscience, 16, 159-182.

Shapiro, K. L., \& Loughlin, C. (1993). The locus of inhibition in the priming of static objects: Object token versus location. Journal of Experimental Psychology: Human Perception \& Performance, 19, 352-363.

SHaw, M. L. (1984). Division of attention among spatial locations: A fundamental difference between detection of letters and detection of luminance increments. In H. Bouma \& D. G. Bowhuis (Eds.), Attention and performance $X$ (pp. 109-121). Hillsdale, NJ: Erlbaum.

Skavenski, A. A., McPeek, R. M., Maljkovic, V., \& Nakayama, K. (1993). Two forms of shortened saccadic eye movement latency in a pop-out visual search task. Society for Neuroscience Abstracts, 19, 426.

SNYDER, C. R. R. (1972). Selection, inspection, and naming in visual search. Journal of Experimental Psychology, 92, 428-431.

SutTER, E. (1992). A deterministic approach to nonlinear systems analysis. In R. B. Pinter \& B. Nabet (Eds.), Nonlinear vision: Determination of neural receptive fields. function, and networks (pp. 171-197). Boca Raton, FL: CRC Press.

TANAKA, Y., \& Shimojo, S. (1993). Location priming and probability priming are isolatable in detection and discrimination. Investigative Ophthalmology \& Visual Science, 33 (Suppl.), 1234.

Tipper, S. P., Brehaut, J. C., \& Driver, J. (1990). Selection of moving and static objects for the control of spatially directed action. Jour- nal of Experimental Psychology: Human Perception \& Performance, 16, $492-504$.

TiPper, S. P., Driver, J., \& Weaver, B. (1991). Object-centered inhibition of return of visual attention. Quarterly Journal of Experimental Psychology: Human Experimental Psychology, 43A, 289-298.

Ttpper, S. P., Weaver, B., Cameron, S., Brehaut, J. C., \& Bastedo, J. (1991). Inhibitory mechanisms of attention in identification and localization tasks: Time course and disruption. Journal of Experimental Psychology: Learning, Memory, \& Cognition, 17, 681-692.

Treisman, A. (1988). Features and objects: The fourteenth Bartlett memorial lecture. Quarterly Journal of Experimental Psychology, 40A, 201-237.

Treisman, A., Vieira, A., \& Hayes, A. (1992). Automaticity and preattentive processing. American Journal of Psychology, 105, 341-362.

Wolfe, J. M., Cave, K. R., \& Franzel, S. L. (1989). Guided search: An alternative to the feature integration model for visual search. Journal of Experimental Psychology: Human Perception \& Performance, $15,419-433$

\section{NOTES}

1. The effects of shape and response are confounded. However, as we were studying the focusing of attention, we were not as interested in the variable that requires attention-that is, shape-purposely chosen not to be very salient (also see Snyder, 1972).

2. Observer E.F.B. has never participated in psychophysical experiments prior to these priming studies.

3. Acuity falls off faster along the vertical than along the horizontal axis (Rovamo \& Virsu, 1979; Kröse \& Julesz, 1989). Using an ellipse equalizes performance for all positions of the target.

4. Whenever observers had to run several thousand trials, we tested them at the rate of 1,000 to 1,400 trials per day over several days. Sometimes these sessions were spread over a period of 2 weeks and sometimes they were held several months apart. Because separate sessions always showed same patterns of results, we collapsed the data over the different periods.

5. In this and all other experiments, all values are means of reaction times for correct trials. Values that fell outside 3 standard deviations were excluded (fewer than $1 \%$ of the trials). Error bars represent standard error of the mean and are, unless otherwise noted, means of all values.

6 . We do not know whether this result is due to the number of repetitions or the time between trials. The duration of a memory trace can also be affected by the trials preceding it and/or following it; that is, through retroactive and proactive inhibition, respectively (Baddeley, 1986). Due to these factors, we cannot claim that the effect of a single trial generally lasts for approximately $30 \mathrm{sec}$, even though it did so in our paradigm. We will report issues relating to time in an upcoming paper.

7. We did not use the condition in which the absolute position of the target remained the same and the relative position changed. Moving the target to change the relative but not the absolute position would have placed the target in the position just occupied by a distractor. As inhibition and facilitation effects would, therefore, cancel each other out, we chose not to employ this condition.

8 . This is the size of priming effects in practiced observers. Naive observers show effects greater than the usual $20-50 \mathrm{msec}$. In initial sessions a single previous trial can prime the current trial by as much as $150 \mathrm{msec}$.

9. The authors, K.N. and V.M., have run many thousands of trials. For K.N. there has been no observed decrement in priming. For V.M. priming apparently did disappear after many thousands of trials, although we found that this loss was due to an asymptotic performance or "floor" effect. Reducing the color difference reestablished the appearance of the priming.

(Manuscript received February 21, 1995; revision accepted for publication January 17, 1996.) 